\title{
Absence of Fluram Bands in Plant Chromosomes
}

\author{
R. Cremonini', D. Pignone ${ }^{2}$, I. Galasso ${ }^{2}$ and N. Colonna ${ }^{3}$ \\ 'Dipartimento di Scienze Botaniche della Università, Via Luca Ghini 5, 56126 Pisa, Italy \\ ${ }^{2}$ Istituto del Germoplasma, CNR, Via Amendola 12570100 Bari, Italy \\ ${ }^{3}$ Scuola Superiore di Studi Universitari e Perfezionamento, \\ Via Carducci 40, 56127 Pisa, Italy
}

Accepted December 28, 1994

By means of different treatments several banding patterns have been demonstrated in the chromosomes of many species, allowing their lengthwise differentiation.

Most of the mechanisms producing differentiation are not always easy to interpret since chromosomes are the result of interaction between nucleic acids and proteins. Sometimes pretreatments provoking chemical or structural alterations of chromosomal organization are necessary in order to get bands, in other cases the banding methods are based only on the affinity of the dye to DNA bases (Sumner 1990 and references therein).

In plants, Giemsa banding techniques allow the cytological detection of heterochromatin, and, therefore, of several types of chromatin concurrently. Fluorochromes, endonucleases and in situ hybridizations point out the DNA characteristics, allowing the detection of single fractions (Schubert 1990, Cremonini 1992).

Recently Cuellar et al. (1991) have described Fluram banding patterns in mammalian chromosomes revealing heterogeneous distribution of chromosomal proteins. Fluram (Fluorescamine: 4-phenylpiro(furane-2(3H), $1^{\prime}$-phtalane)-3-, $3^{\prime}$-dione) is a novel fluorogenic reagent for primary amines in the picomole range (Udenfriend et al. 1972); the Fluram reaction can be an easy method for a primary analysis of different classes of proteins in chromosomes of related species.

Techniques using protein components to produce bands on metaphase chromosomes are very few, and since, to our knowledge up today, there are no data on plant materials using Fluram, in the present paper we report on the experiments carried out on plant chromosomes.

\section{Materials and methods}

Three species were analysed: Vicia faba L., Allium cepa L. and Dasypyrum villosum (L.) Candargy.

Seeds of $V$. faba cv. Aguadulce were soaked in running tap water overnight and germinated in damp vermiculite at $19^{\circ} \mathrm{C}$ until secondary roots were about $1 \mathrm{~cm}$ long.

Seeds of D. villosum, inbred line GHA 01 selected by one of us (D. P.), were soaked in running tap water overnight and placed in Petri dishes on Whatman $\mathrm{n}$. 2 filter paper with $6 \mathrm{ml}$ of deionized water. They germinated in the dark at the temperature of $23^{\circ} \mathrm{C}$ and roots were collected when $1 \mathrm{~cm}$ long. The dishes were sealed with laboratory film in order to avoid partial evaporation.

Roots from bulbs of $A$. cepa cv. Fiorentina, grown at room temperature in distilled water changed twice a day were collected when $1 \mathrm{~cm}$ long.

The roots of the three species were processed with cytostatic and fixative as described below.

Correspondence to: Prof. Roberto Cremonini, Dipartimento di Scienze Botaniche Universita di Pisa, Via Luca Ghini 5, 56126 Pisa, Italy. 
Two different cytostatic treatments were used:

i) colchicine $0.05 \%$ in distilled water for three or five hours,

ii) low temperature obtained immersing the roots in distilled water kept at $0-1^{\circ} \mathrm{C}$ in a ice bath for $12 \mathrm{hr}$.

Four different fixing methods were also applied:

i) Carnoy's fluid (3:1 v/v, ethanol-glacial acetic acid)

ii) Farmer's fluid (1:1 v/v, 95\% ethanol-acetic acid)

iii) $10 \%$ formalin for $24 \mathrm{hr}$

iv) no fixative

The root tips were squashed in a small drop of $45 \%$ acetic acid after a treatment with a $5 \%$ aqueous solution of pectinase (Sigma) for $1 \mathrm{hr}$ at $37^{\circ} \mathrm{C}$ with the addition of $0.001 \mathrm{M}$ EDTA in order to annul the activity of DNase if present (Berlyn et al. 1979) and coverslips removed with the dry-ice tecnique.

Staining with Fluram (Serva) was performed according to Cuellar et al. (1991). Slides were covered with $0.5 \mathrm{ml}$ solution of sodium bicarbonate $(\mathrm{pH} 8.5)$ to which $0.5 \mathrm{ml}$ of a Fluram solution in acetone $(0.5 \mathrm{mg} / \mathrm{ml})$ was added.

Staining was performed for 1 to $5 \mathrm{~min}$; after flushing the excess of dye with water the slides were mounted in a $\mathbf{M c I l v a n e - g l y c e r o l -} \mathbf{M g C l}_{2}$ solution.

In order to assess if different values of $\mathrm{pH}$ may influence the staining properties, sodium bicarbonate batchs adjusted with $\mathrm{NaOH}$ at $\mathrm{pH} 9,10$ and 11 were also tested.

\section{Results and discussion}

Additionally to our experiments on plant materials we have carried out Fluram banding on human lymphocytes according to Cuellar et al. (1991). Human chromosomes showed a bright fluorescence after staining with Fluram as in Cuellar' report. In order to assure the banding capability of our materials, we have also stained some slides with barium saline Giemsa according to Giraldez et al. (1979) after colchicine treatment and fixation with Carnoy's fluid, obtaining the expected banding patterns. The reported method by Fluram staining did not produce any banding pattern in our material, apart from cytostatic treatments, fixatives, staining times or $\mathrm{pH}$ values and all regions of the chromosomes showed a slight and diffuse fluorescence (Fig. 1).

According to Udenfriend et al. (1972) and Lei (1977) the unique groups that can be expected to be revealed in chromatin after Fluram treatment are lysine residues from proteins since aliphatic and unsubstituted amines originate the fluorescent product.

Cuellar et al. (1991) in mouse and human metaphase chromosomes found a difference in the protein reactivity: mouse centromeric heterochromatin is highly reactive, showing intense fluorescence in all centromeric regions, whereas human chromosomes show fluorescence in pericentromeric regions and no fluorescence in centromeric ones. A G-like banding pattern is also obtained in both materials. Therefore they suggest that proteins in G-banded regions are more conserved. G-bands can be demonstrated easily and regularly on chromosomes of higher vertebrates but only sporadically in other animals and in plants.

Giemsa banding is generally held to show no DNA base specificity and explanations of G-banding mechanism have been based on DNA-proteins interactions and not on properties of DNA alone.

In plants, according to Greilhuber (1977), metaphase chromosomes do not show G-bands because they are much more contracted than those of mammals. A wide vision on the nature of positive and negative $G$ bands is reported by Sumner (1990), but at present, still there is no satisfactory hypothesis to explain the mechanism of G-banding and the absence of G-bands in 


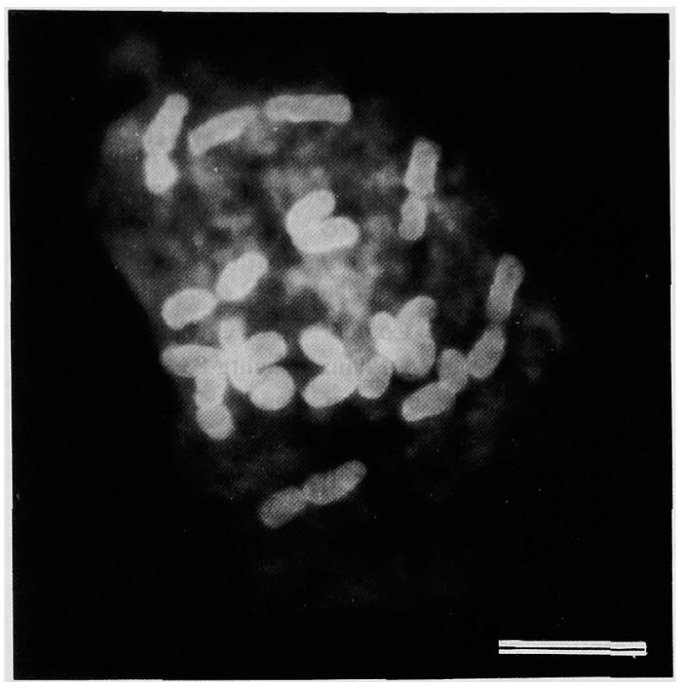

Fig. 1. Metaphase of Dasypyrum villosum stained with Fluram. Bar represents $10 \mu \mathrm{m}$.

plant material.

Therefore the absence of Fluram banding in our material might be due to the incapability of Fluram of doing bonds with plant chromosomes owing to their different organization between DNA and chromosomal proteins.

Moreover Hadlaczky et al. (1989) have demonstrated in mouse and human chromosomes the existence of a mitosis specific centromere antigen. This protein can be found exclusively in mitotic chromosomes and its presence is necessary for the active function of centromere. This protein has not yet been demonstrated to be involved in Fluram banding; nor, up-to-date, has it been demonstrated to be present in plant chromosomes. Nevertheless, it is interesting to point out that bright Fluram bands have been observed in organisms possessing this protien and have not observed in organisms likely missing it. This might indicate a more substantial role of this antigen and of other specific chromosomal proteins in the Fluram expression.

\section{Summary}

Longitudinal differentiation of metaphase chromosomes has been investigated using several techniques. Recently Cuellar et al. (1991) using Fluram induced species dependent $\mathrm{C}$ and G-bands in mammaliam chromosomes revealing heterogeneous distribution of chromosomal proteins.

Metaphase chromosomes from root tips of three plant species as Vicia faba, Allium cepa and Dasypyrum villosum were tested by Fluram in order to verify if, in plant, is possible to obtain Fluram banding. No banding pattern was observed and all regions of the chromosomes showed a slight and diffuse fluorescence.

\section{Acknowledgement}

This work was partially supported by a grant from M. U. R. S. T. and from C. N. R. (94.01025.CT06), Rome. 


\section{References}

Berlyn, G. P., Dhillon, S. S. and Miksche, J. P. 1979. Feulgen cytophotometry of pine nuclei. II: Effect of pectinas used in cell separation. Stain Technol. 54: 201-204.

Cuellar, T., Gosalvez, J., Del Castillo, P. and Stockert, J. C. 1991. Fluram induces species-dependent C and G band in mammalian chromosomes, revealing heterogeneous distribution of chromosomal proteins. Genome 34 $772-776$.

Cremonini, R. 1992. The chromosomes of Vicia faba: banding pattern and in situ hybridization. Biol. Zentbl. 111 188-203.

Giraldez, R., Cermeno, M. C. and Orellana, J. 1979. Comparison of C-banding pattern in the chromosomes of inbre lines and open pollinated varieties of rye. Z. Pflanzenzuchtg 83: 40-48.

Greilhuber, J. 1977. Why plant chromosomes do not show G-bands? Theor. Appl. Genet. 50: 121-124.

Hadlaczky, G., Praznovszky, T., Rasko, I. and Kereso, J. 1989. Centromere proteins. I. Mitosis specific centromer antigen recognized by anti-centromere autoantibodies. Chromosoma 97: 282-288.

Lai, C. Y. 1977. Detection of peptides by fluorescence methods. Methods Enzymol. 47: 236-243.

Schubert, I. 1990. Restriction endonucleases (RE-) banding of plant chromosomes. Caryologia 43: 117-130.

Sumner, A. T. 1990. Chromosome banding. Unwin Hyman, London.

Udenfriend, S., Stein, S., Bohlen, P., Dairman, W., Leimgruber, W. and Wiegele, M. 1972. Fluorescamine: a reagen for assay of amino acids, peptides, proteins and primary amines in the picomole range. Science 178: $871-872$ 